\title{
Propranolol-induced Somnambulism: A Case-report in a Patient with Essential Tremor
}

\author{
Niem Tu Huynh, Philippe Huot
}

Keywords: Movement disorders, sleep

doi:10.1017/cjn.2014.101

Can J Neurol Sci. 2014; 41: 787-788

Essential tremor is a common neurological disorder characterised by postural and kinetic tremor. In the American Academy of Neurology 2011 evidence-based guidelines for the treatment of essential tremor, propranolol and primidone were the only two medications for which Level A recommendations were issued $^{1}$. Both medications are usually well tolerated. We recently prescribed propranolol to a 34-year old woman with essential tremor to alleviate her symptoms and encountered a rare adverse effect, somnambulism. We report the case here and perform a brief review of the literature on beta-blocker-induced sleep disturbance.

\section{Case Report}

A woman in her mid-thirties was seen at the movement disorder clinic for incapacitating tremor. Besides tremor, her past medical history was negative. In particular, there was no history even as a child, of parasomnias such as night terrors, somnambulism or somniloquy. She was not taking any medications that could have caused tremor, such as valproic acid, lithium, bronchodilators, etc. When she was seen, she had been presenting tremor for slightly less than a decade. Tremor severity progressively increased, which led her to seek medical opinion. Tremor description was typical of essential tremor, i.e. it affected both upper limbs, was postural and kinetic, improved by alcohol and interfered with her ability to eat soup with one hand or drink from a cup with one hand. Her family history was positive for tremor, as her maternal grand-father presented tremor which, according to the patient, was similar to hers.

Upon examination, the tremor affected both upper limbs, without evidence of lateralisation, was postural and kinetic, of high frequency and low amplitude. The pour-test was impaired. There was no head, tongue, voice or lower limb tremor. There was no resting component and no evidence of bradykinesia or rigidity. Tandem gait was slightly impaired, as the woman had increased body sway, without losing balance. The remainder of the neurological examination was normal, without evidence of cerebellar dysfunction or neuropathy. Brain computed tomography (CT) scan and thyroid function tests were normal.

Propranolol was commenced without titration at $20 \mathrm{mg}$ thrice daily (with meals). Tremor was improved by about $50 \%$ after a few doses. Approximately two weeks after being started on propranolol, she began experiencing episodes of somnambulism. The sleep walking episodes would occur once or twice a week, exclusively at night time, at different times during the night. Typical duration ranged from 5 to 30 minutes. She would not wake up during the episodes and had no recollection of them. The episodes consisted of walking purposelessly in the house, without talking or interacting with her partner. On rare occasions, she would also exhibit somniloquy. There was no evidence of other parasomnia, such as sleep paralysis, hallucinations, dream enactment, cataplexy, etc. She continued taking propranolol for about one month after the episodes of sleep walking started and then discontinued the medication because she was afraid she might get hurt or hurt her partner during an episode. The episodes of somnambulism did not recur after propranolol was discontinued. However, tremor re-emerged and the patient was started on primidone, which did not trigger sleep walking.

\section{DISCUSSION}

Beta-blocker-induced sleep disturbance and nightmares are well-described phenomena that are more frequent when lipophilic drugs, such as propranolol are used, as these agents readily penetrate the brain $^{2}$. Although the mechanisms underlying beta-blockerinduced sleep alterations remain to be elucidated, reduced melatonin levels may be involved ${ }^{2}$ and administration of melatonin might attenuate parasomnias in patients taking beta-blockers ${ }^{3}$.

Although beta-blocker-induced sleep alterations are common, beta-blocker-induced sleep walking appears to be a rare complication. To the best of our knowledge, only four cases of somnambulism in patients taking beta-blockers have been reported. Three of these patients were on propranolol ${ }^{4}$, whereas one was on metoprolol ${ }^{5}$. All of these patients previously had experienced somnambulism during their childhood, which is in contrast to our patient, who had not experienced sleep walking during her early years. The three patients who experienced somnambulism while on propranolol were on total daily doses ranging from 80 to $120 \mathrm{mg}$, which is higher than the dose of the patient reported here. As these three patients were taking propranolol for migraine prophylaxis, it

From the Espera Neuroscience Inc Montreal, Quebec (NTH, PH); Department of Pharmacology (PH), Division of Neurology (PH), Faculty of Medicine, Université de Montréal and Centre Hospitalier de l'Université de Montréal, Montréal, Quebec, Canada. Received May 30, 2014. Final Revisions Submitted October 2, 2014. Correspondence to: Philippe Huot, Centre Hospitalier de l'Université de Montréal, 1560 Sherbrooke East Street, Deschamps Pavilion GR-1177, Montréal, QC, Canada, H2L 4M1. Email: p.huot@umontreal.ca 
was suggested that propranolol would trigger somnambulism only under two conditions, 1) patients had previously experienced sleep walking and 2) patients had migraines, and the authors postulated that there might be a link between migraines and sleep walking ${ }^{4}$. This potential link between migraines and somnambulism induced by beta-blockers was challenged by a case-report study in which metoprolol induced sleep walking in woman without history of migraine, but who had experienced sleep walking during childhood $^{5}$.

The case we present is therefore the first one in which a woman who had never experienced somnambulism in childhood developed sleep walking while taking propranolol. As such, it would seem that previous somnambulism is not a required condition to experience somnambulism under propranolol treatment. Whether other betablockers may also elicit somnambulism in patients who have never presented the condition remains unknown. Equally unknown is whether propranolol and other beta adrenoceptor antagonists may also elicit somnambulism in men, as all of the cases reported so far, including ours, occurred in women. Moreover, the case reported here is also the one in which the lowest daily dose of propranolol, $60 \mathrm{mg}$ daily, induced somnambulism. Whether doses of propranolol lower than $60 \mathrm{mg}$ daily would be sufficient to elicit somnambulism is unknown.

\section{ACKNOWLEDGEMENTS}

This work was supported by the Department of Pharmacology and Faculty of Medicine of Université de Montréal and by Espera Neuroscience Inc.

\section{Disclosures}

There are no conflicts of interest to declare. $\mathrm{PH}$ holds an equity position in Espera Neuroscience Inc.

\section{REFERENCES}

1. Zesiewicz TA, Elble RJ, Louis ED, et al. Evidence-based guideline update: treatment of essential tremor: report of the Quality Standards subcommittee of the American Academy of Neurology. Neurology. 2011;77:1752-5.

2. Brismar K, Hylander B, Eliasson K, Rossner S, Wetterberg L. Melatonin secretion related to side-effects of beta-blockers from the central nervous system. Acta Med Scand. 1988;223:525-30.

3. Fares A. Night-time exogenous melatonin administration may be a beneficial treatment for sleeping disorders in beta blocker patients. J Cardiovasc Dis Res. 2011;2:153-5.

4. Pradalier A, Giroud M, Dry J. Somnambulism, migraine and propranolol. Headache. 1987;27:143-5.

5. Hensel J, Pillmann F. Late-life somnambulism after therapy with metoprolol. Clin Neuropharmacol. 2008;31:248-50. 